\title{
CORRELAÇÕES DENDROCLIMATOLÓGICAS DO Eucalyptus grandis Hill ex Maiden NA REGIÃO DE RIO CLARO, RJ
}

\author{
DENDROCLIMATOLOGY CORRELATIONS OF Eucalyptus grandis Hill ex Maiden OF RIO CLARO, \\ RJ STATE - BRAZIL
}

\author{
Bruna Roque Ugulino de Oliveira ${ }^{1}$ João Vicente de Figueiredo Latorraca ${ }^{2}$ \\ Mário Tomazello Filho ${ }^{3}$ Rosilei Aparecida Garcia ${ }^{4}$ Alexandre Monteiro de Carvalho 5
}

\begin{abstract}
RESUMO
O plantio de florestas comerciais contribui decisivamente na geração de benefícios socioeconômicos e ambientais, conferindo caráter de sustentabilidade aos empreendimentos de base florestal. O Eucalyptus grandis Hill Ex Maiden se destaca por ser uma das espécies mais cultivadas em reflorestamentos no Brasil. O objetivo deste estudo foi determinar as correlações entre a precipitação e a produção da madeira de Eucalyptus grandis com 23 anos de idade provenientes de um plantio comercial no município de Rio Claro, RJ. O incremento anual da madeira foi determinado através da técnica da densitometria de raios $\mathrm{X}$, a qual permitiu a obtenção de um perfil detalhado da densidade na direção radial para cada amostra. Considerou-se como um ano de vida da árvore, o período decorrido para a formação de dois anéis outonais consecutivos, ou seja, dois picos de alta densidade (REZENDE e FERRAZ, 1985 e FERRAZ, 1993). Os resultados mostraram uma correlação positiva entre os dados de precipitação e o incremento anual da madeira, denotando o potencial dendroclimatológico da espécie nessa Região.
\end{abstract}

Palavras-chave: anéis de crescimento; densitometria de raios X; precipitação.

\begin{abstract}
The commercial forests plantation contributes decisively to generate socio-economic and environmental benefits, providing sustainability at the forest-based industries. The Eucalyptus grandis Hill ex Maiden stand out as one of the most cultivated species for reforestation in Brazil. The objective of this study was to determine the relationship between precipitation and wood production for 23-year old Eucalyptus grandis from a commercial plantation in Rio Claro, Rio de Janeiro state, Brazil. The annual increment of wood was determined by the X-ray densitometry, which provides a detailed wood density profile in radial direction for each sample. One year of the tree life was considered because this the period for the formation of two consecutive latewood rings formation, which corresponds to two high density peaks. The results showed a positive correlation between precipitation data and annual increment of wood, revealing the dendroclimatology potential of this species in this region.
\end{abstract}

Keywords: precipitation; tree rings; x-ray densitometry.

1. Engenheira Florestal, Mestranda em Ciências Ambientais e Florestais, Universidade Federal Rural do Rio de Janeiro, BR 465, Km 07, CEP 23890-000, Seropédica (RJ). braugulino@msn.com

2. Engenheiro Florestal, Dr., Professor da Universidade Federal Rural do Rio de Janeiro, BR 465, Km 07, CEP 23890-000, Seropédica(RJ). latorraca@ufrrj.br

3. Engenheiro Agrônomo, Dr., Professor do Departamento de Ciências Florestais, Escola Superior de Agricultura Luiz de Queiroz, Universidade de São Paulo, Av. Pádua Dias, 11, Caixa Postal 09, CEP 13418-900, Piracicaba (SP).mtomazel@esalq.usp.br

4. Engenheira Florestal, Dra ${ }^{\mathrm{a}}$, Professora da Universidade Federal Rural do Rio de Janeiro, BR 465, Km 07, CEP 23890-000, Seropédica (RJ). rosileigar@ufrrj.br

5. Engenheiro Florestal, Dr. Professor, Universidade Federal Rural do Rio de Janeiro, BR 465, Km 07, CEP 23890-000, Seropédica (RJ). amcarvalho@ufrrj.br

Recebido para publicação em 28/08/2009 e aceito em 25/08/2010

Ci. Fl., v. 21, n. 3, jul.-set., 2011 


\section{INTRODUÇÃO}

O Eucalyptus grandis é uma das espécies mais dinâmicas e indicadas para usos múltiplos, tendo em vista as altas taxas de produtividade das florestas plantadas, boa adaptação em grande parte do território brasileiro e disponibilidade considerável de florestas para pronto uso (SILVA, 2002). A crescente busca pela destinação do uso da madeira de Eucalyptus para fins mais nobres, como fabricação de móveis e construção civil, resulta em agregação do valor dos produtos madeireiros e madeiras de melhor qualidade. Dessa forma, para a adequada aplicabilidade da madeira é importante determinar as suas características anatômicas, físicas, químicas e mecânicas exigidas para a produção de produtos específicos. Estas propriedades, por sua vez, estão intimamente relacionadas com as atividades cambiais, as quais sofrem influência direta das condições ambientais onde a planta se desenvolve.

A densidade da madeira destaca-se, entre os parâmetros utilizados na qualificação e usos da madeira, por fornecer inúmeras informações sobre as suas características. Da densidade depende a maior parte das demais propriedades físicas e tecnológicas da madeira. Entre os métodos de determinação de densidade da madeira destaca-se o da densitometria de raios $\mathrm{X}$, o qual permite determinar a distribuição da densidade ao longo das microvariações da estrutura anatômica da madeira (OLIVEIRA et al., 2005; ROQUE, 2005). Dentre as vantagens do uso de dados densitométricos obtidos pela metodologia de raios $\mathrm{X}$ em estudos, destaca-se a visualização e a demarcação dos anéis anuais através do perfil de densidade. Segundo Tomazello Filho (2006), a análise de densitometria de raios $\mathrm{X}$ em amostras de madeira possibilita medir a intensidade de raios que atravessam o lenho destas e com o uso de programas específicos, a obtenção de um perfil da densidade na direção radial da madeira. Segundo o mesmo autor, através do perfil detalhado da densidade na direção radial da madeira, os limites dos anéis de crescimento podem ser visualizados. A partir da delimitação das camadas de crescimento, pode-se determinar o incremento anual. O incremento anual pode ser correlacionado com as médias mensais ou totais anuais de precipitação do local de coleta das amostras e dessa forma, verificar o potencial dendroclimatológico da espécie.

A dendroclimatologia baseia-se no princípio de extração de registros das variáveis climáticas gravadas nos anéis de crescimento ou camadas de crescimento. Nos últimos anos, estudos desses anéis em árvores estão sendo usados para determinar ou verificar fatores climáticos que prevalecem em um dado lugar ou região que possam causar variações na dinâmica de crescimento de árvores, e na sua qualidade (TROVATI e FERRAZ, 1984; RIGOZO, 1999; RIGOZO et al., 2004; e RASPOPOV et al., 2004). Entretanto são poucos os estudos sobre os efeitos de fatores climáticos no incremento anual em madeiras com anéis pouco evidentes, como é o caso do Eucalyptus grandis. A importância da determinação da densidade está na sua estreita relação com a qualidade da madeira e na correlação dos picos de densidade com o seu incremento anual.

$\mathrm{O}$ presente trabalho teve por objetivo determinar as correlações entre a precipitação e a produção da madeira de Eucalyptus grandis proveniente de um plantio comercial no município de Rio Claro, RJ, a partir da análise do perfil de densidade da madeira e dessa forma contribuir com os estudos de dinâmica de crescimento dessa espécie.

\section{MATERIAL E MÉTODO}

\section{Local de estudo e coleta do material}

O material utilizado neste estudo foi obtido de um plantio comercial de Eucalyptus grandis Hill ex Maiden, com idade de 23 anos, localizado no distrito de Passa Três, município de Rio Claro no Estado do Rio de Janeiro. O clima da região é do tipo Ws (Köppen) com estação chuvosa no verão e seca no inverno, com temperatura média máxima de $24^{\circ} \mathrm{C}$ nos meses de janeiro e fevereiro, e temperatura média mínima de $16.7^{\circ} \mathrm{C}$ em julho. Inicialmente, foram selecionadas aleatoriamente seis árvores, num raio de 50 metros entre elas, as quais tiveram discos de $40 \mathrm{~cm}$ de altura retirados a $1.30 \mathrm{~m}$ (altura 1 ou altura do DAP) e $3.60 \mathrm{~m}$ do solo (altura 2).

\section{Preparo das amostras \\ Obtenção das seções transversais}

Os discos foram levados ao Laboratório de Usinagem e Processamento da Madeira do Departamento de Produtos Florestais, do Instituto de Florestas da UFRRJ para seccionamento e obtenção de seções transversais das amostras, devidamente identificadas. Estes discos tiveram uma altura inicial de $40 \mathrm{~cm}$, a qual foi reduzida a $10 \mathrm{~cm}$ e foram cortadas suas costaneiras. Foi traçado nos discos uma linha passando pela medula e a partir dela

Ci. Fl., v. 21, n. 3, jul.-set., 2011 
delimitada a largura final da seção transversal de 10 $\mathrm{mm}$. As amostras utilizadas para a densitometria de raios X são ilustradas na Figura 1.

Posteriormente, as seções diametrais de 10 $\mathrm{mm}$ de largura e $10 \mathrm{~cm}$ de altura, foram cortadas no sentido transversal da madeira em $2 \mathrm{~mm}$ de espessura (altura) com um equipamento de dupla serra circular paralela disponível no Laboratório de Anéis de Crescimento e Densitometria de Raios X do Departamento de Ciências Florestais da ESALQ/ USP.

\section{Obtenção das radiografias de raios $\mathrm{X}$}

$A$ análise de raios $\mathrm{X}$ foi realizada nas dependências do Laboratório de Anéis de Crescimento e Densitometria de Raios X, do Departamento de Ciências Florestais da ESALQ/ USP de acordo com a técnica descrita por Amaral (1994). Para que as amostras fossem radiografadas, estas precisavam atingir um teor de umidade de equilíbrio de aproximadamente 12\%. Dessa forma, as amostras foram levadas para a sala de raios $\mathrm{X}$, sob condições de temperatura e umidade controladas $\left(20^{\circ} \mathrm{C}, 65 \%\right.$ UR) por um período de aproximadamente 12 horas. Após este período, as amostras foram dispostas sobre uma camada da mesa de acetato de celulose contendo uma escala cinza, e coberta por outra camada de acetato de celulose. O filme radiográfico foi colocado sob a camada móvel da mesa de acetato em condições de total ausência de luz.

As amostras foram radiografadas em equipamento de raios $\mathrm{X}$ da Hewlett Packard, modelo Faxitron 43805 N, a uma distância da fonte de raios X e o filme radiográfico de $120 \mathrm{~cm}$, por um tempo de exposição de 5 minutos. O filme utilizado foi o Kodak Diagnostc Film X-Omat XK1, com dimensões de $24 \times 18 \mathrm{~cm}$.

Os filmes radiográficos depois de revelados foram digitalizados em scanner Hewllett Packard ScanJet 6100C/T a uma resolução de 1000 dpi (pixel por polegada) em escala de cinza de 256 graus.

\section{Análise dos filmes radiográficos}

Para a análise dos filmes radiográficos, foram utilizados dois softwares: Crad 1.04 e Cerd 2.08. No software Crad, utilizou-se a imagem digitalizada dos filmes para selecionar a amostra e a partir dela realizar as comparações entre a escala de cinza, que possui valores conhecidos de densidade, e as amostras radiografadas. Os valores de densidade foram determinados com o auxílio do software Cerd a cada $500 \times 10 \mu \mathrm{m}$ (comprimento x largura) para cada segmento (anel de crescimento) das amostras do lenho. Através das planilhas eletrônicas foram obtidos os perfis de densidade das amostras.

Os dados para cada árvore foram dispostos em dois raios (Raio 1 à esquerda e Raio 2 à direita do gráfico) e assim obteve-se um gráfico com os valores de densidade (eixo das ordenadas) para cada distância considerada na delimitação dos anéis de crescimento (eixo das abscissas). As amostras diametrais foram medidas ao longo de sua largura com paquímetro eletrônico, para que correções fossem feitas caso sua largura não apresentasse 2 $\mathrm{mm}$ de espessura e, dessa forma, poder alterar os valores de densidade devido a uma maior ou menor espessura. As correções foram feitas utilizando-
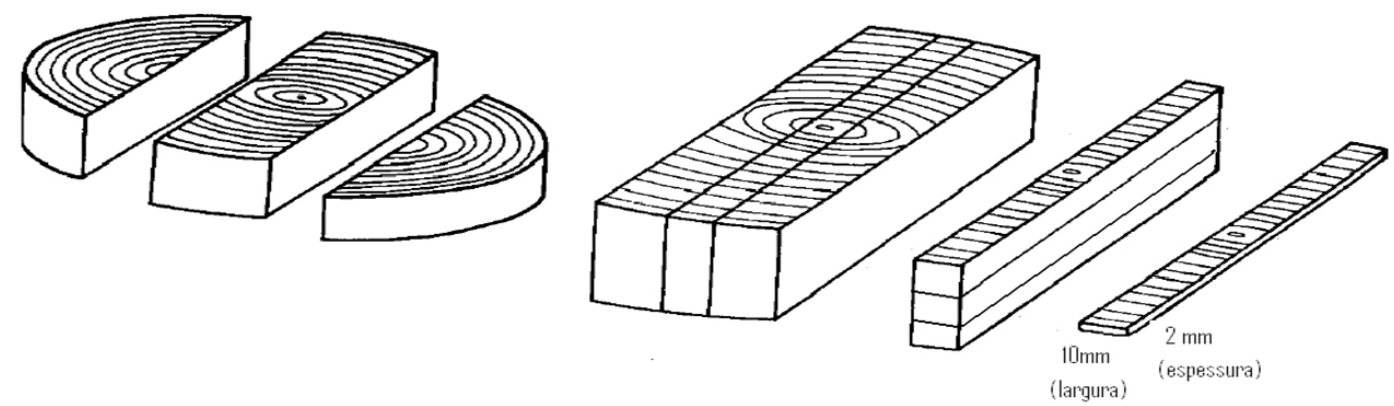

Densitometria de

raio $x$

FIGURA 1: Preparo das amostras do lenho de eucalipto para análise por densitometria de raios X (adaptado de Roque, 2005).

FIGURE 1: Preparation of samples from eucalyptus wood disks for X-ray densitometry analysis (adapted from Roque, 2005). 
se a razão entre a espessura de $2 \mathrm{~mm}$ sobre a espessura real ( $2 \mathrm{~mm} /$ espessura real), sendo esse fator multiplicado pela densidade correspondente na amostra.

\section{Obtenções dos dados climáticos}

As séries históricas de precipitação foram obtidas da Estação Principal Meteorológica de Lídice, disponível no site da Agência Nacional de Águas (ANA), distrito do município de Rio Claro, RJ. Utilizou-se dados de precipitação entre o período de Janeiro de 1985 a Dezembro de 2003 para a correlação com o incremento anual, correspondente aos anéis de crescimento do mesmo período.

\section{Delimitação dos anéis de crescimento}

Os anéis anuais foram identificados através do perfil de densidade nos dois raios de cada amostra, obtidos através da técnica de raios $\mathrm{X}$. Geralmente, os eucaliptos apresentam anéis anuais pouco evidentes, dificultando a determinação dos períodos de crescimento. Por esta razão, para a determinação de um ano de vida da árvore, foi considerada a formação de dois picos consecutivos de alta densidade, como o final do lenho tardio, mesma metodologia usada por Rezende e Ferraz (1985) e Ferraz (1993) em trabalhos similares. Para a correlação com os dados de precipitação foram utilizadas as informações de incremento anual do raio que apresentou um maior número de anéis para cada amostra.

\section{Análise Estatística}

As análises estatísticas foram feitas utilizando-se o software Sistema para Análises Estatísticas (SAEG versão 9.1, ano 2007) desenvolvido pela Universidade Federal de ViçosaMG. Verificou-se se havia correlação de Pearson entre o incremento anual e os dados de precipitação.

\section{RESULTADOS E DISCUSSÃO}

\section{Perfil de densidade}

A técnica de densitometria de raios $\mathrm{X}$ possibilitou a análise precisa das variações radiais da densidade aparente do lenho de árvores de Eucalyptus grandis. Os resultados dos perfis radiais de densidade do lenho das 6 árvores de eucalipto nas duas alturas são apresentados na Figura 2 e Figura 3 indicando um aumento da densidade do lenho no sentido medula-câmbio. As variações de densidade foram significativas, com valores entre $0.3-0.4 \mathrm{~g} / \mathrm{cm}^{3}$ na região próxima à medula $\mathrm{e}$ 0.8-0.9 $\mathrm{g} / \mathrm{cm}^{3}$ de densidade na região próxima ao câmbio para a altura 1 , e com valores entre $0.4-0.5$ $\mathrm{g} / \mathrm{cm}^{3}$ na região próxima à medula e $0.7-0.8 \mathrm{~g} / \mathrm{cm}^{3}$ de densidade na região próxima ao câmbio para a altura 2, sendo que esse comportamento da variação radial tem sido comumente observado para o lenho de eucalipto e de outras espécies florestais. Vários
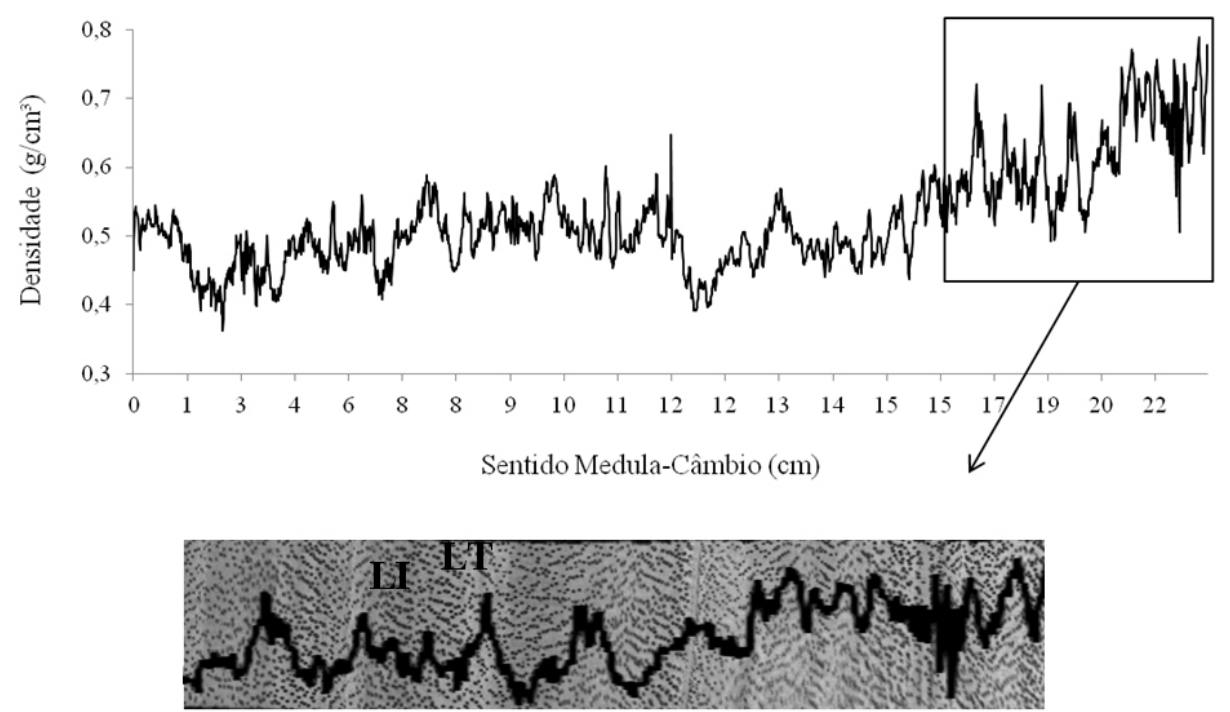

FIGURA 2: Exemplo de um perfil de densidade e imagem macroscópica do lenho radiografado indicando as variações intra e interanual (LI-Lenho Inicial, LT-Lenho Tardio).

FIGURE 2: Example for density profile and X-ray macroscopic image of wood showing intra and interannual variations (LI-earlywood, LT-latewood). 

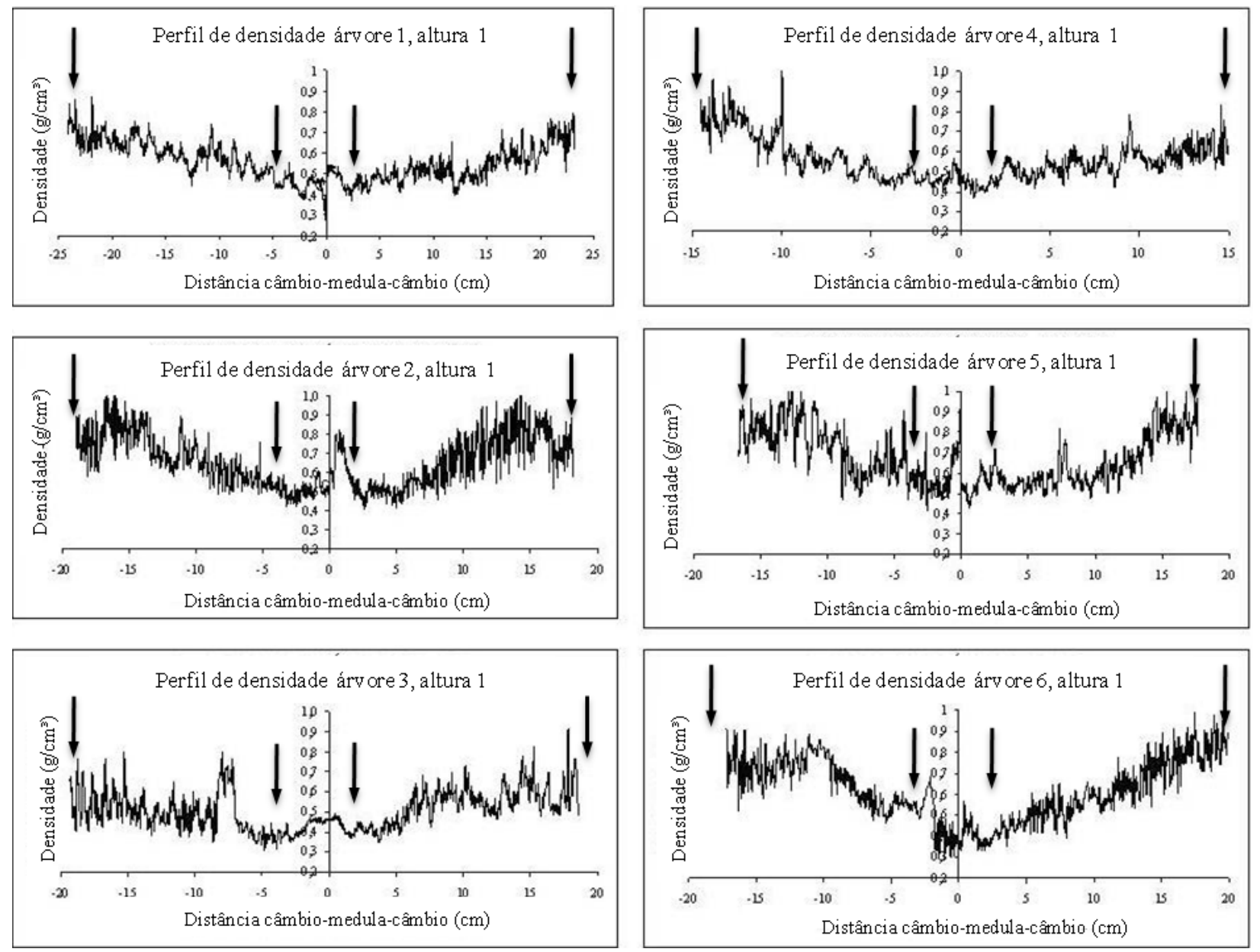

FIGURA 3: Perfis de densidade das amostras de madeira de Eucalyptus grandis no sentido câmbio-medulacâmbio na altura 1(1,30 m do solo), onde as setas indicam os valores máximos e mínimos de densidade.

FIGURE 3: Density profiles of Eucalyptus grandis wood samples from cambium to pith at $1.30 \mathrm{~m}$ height where the arrows indicate the maximum and minimum density values.

autores, como Coutinho (1984), Ferreira (1970), Foelkel et al. (1983), Oliveira et al. (2005), Rezende e Ferraz (1985), Tomazello $(1985,2006)$ e Vital et al. (1987) encontraram para o gênero de Eucalyptus, resultados coerentes com essa tendência. Segundo Ribeiro e Zani Filho (1993) o aumento da densidade da madeira de eucalipto irá variar com a idade, com tendência de estabilização após a formação da madeira adulta. Enquanto que, para o modelo de variação com a altura da árvore, a densidade não seguiu o resultado esperado conforme demais resultados encontrados para a madeira de Eucalyptus na literatura. Resultados estes como o de Rezende et al. (1998) ao estudarem a variação da densidade da madeira de Eucalyptus grandis, aos oito anos, observaram que os valores encontrados na base $(0 \%)$ foram significativamente maiores do que os encontrados a $25 \%$ da altura comercial do tronco.
Porém, Panshin e Zeeuw (1970), discorreram em seu estudo sobre a existência de três tendências de variação da densidade de acordo com a altura da árvore. Segundo os autores, a densidade pode diminuir uniformemente com a elevação da altura no tronco, diminuir na parte inferior do tronco e depois haver um acréscimo regular para a parte superior ou ainda aumentar da base para o topo de forma não uniforme.

As variações intra e interanual de densidade indicou a formação de zonas fibrosas, com maior porcentagem de fibras de parede espessa e menor porcentagem de vasos, visualizável através dos altos picos de densidade ao longo das amostras, conforme a Figura 2 as variações de densidade indicaram 2 diferentes tipos de lenho no tronco das árvores de eucalipto, sendo a madeira juvenil (interna) e adulta (externa). As dimensões das fibras variaram de 
maneira diferente, em relação à posição em altura do tronco das árvores estudadas.

Observando-se as Figuras 3 e 4, pode-se visualizar que as amostras da altura 2 tendem a apresentar maior porcentagem de fibras de parede espessa e menor porcentagem de vasos que as amostras da altura 1 .

A presença de picos de alta densidade, os quais se sobressaem de forma anormal entre os demais, em alguns perfis de densidade, indica a presença de defeitos na madeira e o possível acúmulo de extrativos. As variações na densidade da madeira, entre e dentro de indivíduos da mesma espécie, estão relacionadas ao resultado diferenciado no volume de poros e na presença de extrativos (PALERMO, 2003). Segundo Tomazello Filho et al. (2001), as árvores mostram uma reação às variáveis ambientais que afetam seus inúmeros processos fisiológicos, tais como respiração, fluxo
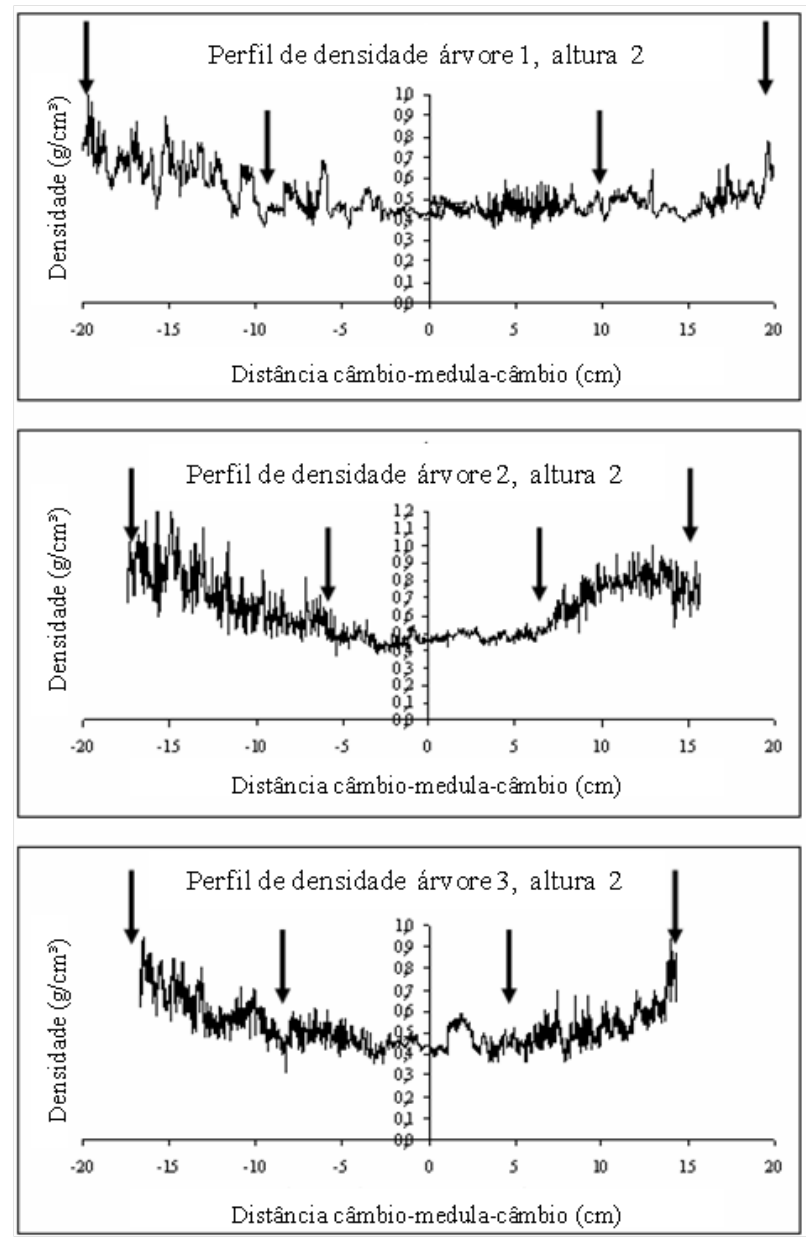

de seiva, transpiração, taxa de divisões celulares entre outros fatores, refletindo na atividade cambial e na anatomia do lenho.

\section{Delimitação dos anéis de crescimento}

Foram utilizadas apenas as amostras das árvores 1,4 e 5 para a identificação das camadas anuais, pois estas apresentaram a demarcação de anéis visíveis. Dessa forma a obtenção do incremento anual foi feita a partir da delimitação dos anéis no perfil de densidade. A data de plantio indica que as árvores possuem 23 anos. Considerando as amostras das árvores na altura 1 (altura do DAP), a árvore 1 apresentou 23 camadas anuais em ambos os raios. Enquanto na altura 2 (altura de $3.60 \mathrm{~m}$ do solo) para a mesma árvore, foram encontradas 19 camadas anuais. De uma forma geral, a idade final da árvore será o número de anéis de crescimento contados na altura do DAP
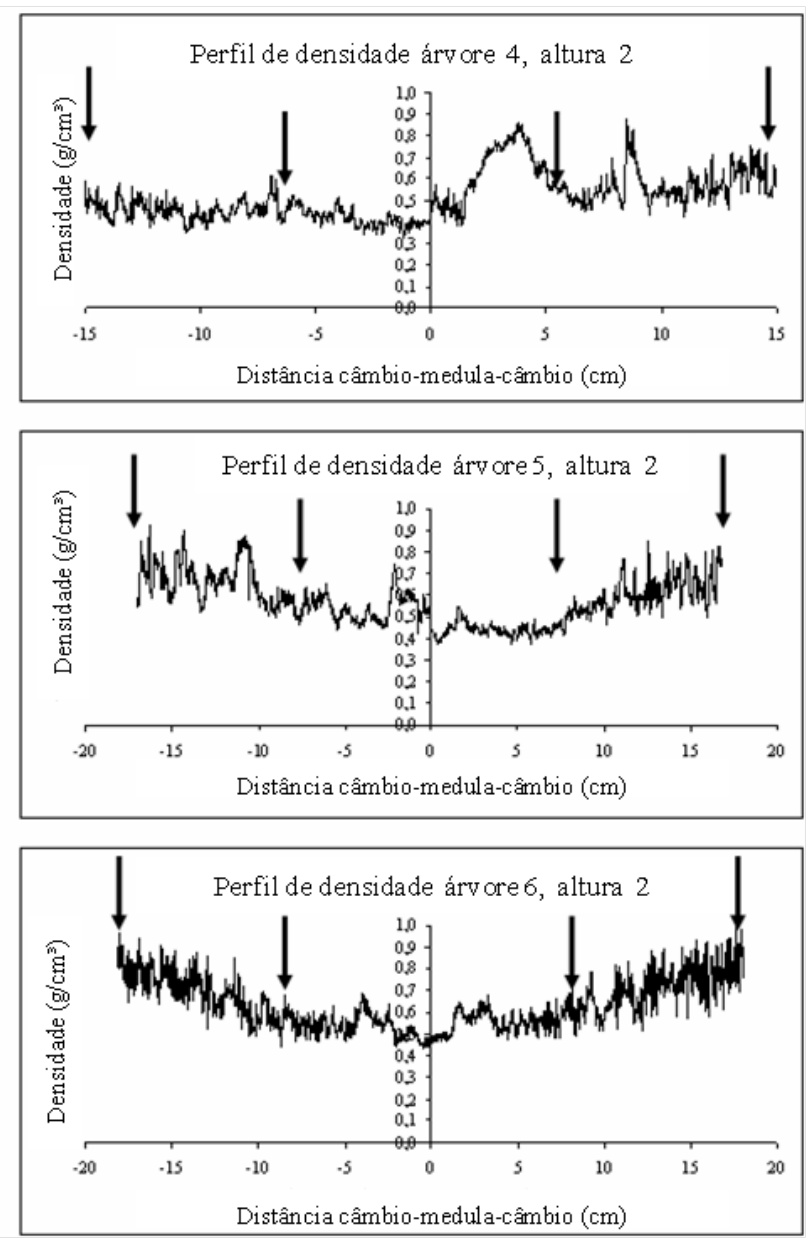

FIGURA 4: Perfis de densidade das amostras de madeira de Eucalyptus grandis no sentido câmbio-medulacâmbio na altura 2 (3,60 m do solo), onde as setas indicam os valores máximos e mínimos de densidade.

FIGURE 4: Density profiles of Eucalyptus grandis wood samples from cambium to pith at $3.6 \mathrm{~m}$ height, the arrows indicate the maximum and minimum density values. 
mais a idade necessária para a árvore atingir 1.30 metros de altura (ENCINAS et al., 2005). Isto é devido ao fato dos anéis de crescimento se estender na forma de cones longitudinalmente. Sendo assim, uma tora apresentará mais anéis na base do que no topo de uma árvore. $O$ fato da árvore 1 na altura do DAP ter apresentado o mesmo número de anéis do que a idade conhecida, 23 anos, indica que o crescimento em altura dessa árvore no primeiro ano ocorreu acima de $1.30 \mathrm{~m}$. Coutinho et al. (2004) ao avaliar o comportamento de espécies de eucalipto na Zona da Mata Pernambucana, encontrou para 18 plantas da espécie Eucalyptus grandis uma altura média de 5.85 m após 12 meses. Rezende e Ferraz (1986), estudando árvores de Eucalyptus grandis provenientes de um plantio comercial na cidade de Salto, no Estado de São Paulo, ao determinarem os perfis de densidade através do uso da radiação gama na altura do DAP também encontraram, através dos mesmos, um número de anéis correspondente à idade conhecida do povoamento, 13 anos. Resultados estes que corroboram com o encontrado no presente estudo. A árvore 4 apresentou 20 e 19 camadas anuais respectivamente nas alturas 1 e 2 . Enquanto a árvore 5 apresentou 22 e 20 camadas anuais, respectivamente, nas alturas 1 e 2 . Foi adotado o número mínimo de camadas encontradas em todas as árvores, 19, para serem feitas a verificação das correlações com os dados de precipitação.

\section{Correlação entre precipitação e incremento anual em diâmetro}

$\mathrm{Na}$ Figura 5, visualizam-se os valores de incremento por ano das árvores e os valores de precipitação total anual. Na Tabela 1, são apresentadas as correlações existentes entre 0 incremento anual em diâmetro $(\mathrm{cm})$ e a precipitação total anual (mm).

TABELA 1: Correlação entre os incrementos anuais e os dados de precipitação total anual.

TABLE 1: Correlation between annual increments and total annual precipitation.

\begin{tabular}{cccc}
\hline Árvore & Altura & Observações & Correlação \\
\hline 1 & 1 & 19 & 0,7449 \\
1 & 2 & 19 & 0,7275 \\
4 & 1 & 19 & 0,5552 \\
4 & 2 & 19 & 0,4698 \\
5 & 1 & 19 & 0,6854 \\
5 & 2 & 19 & 0,6982 \\
\hline
\end{tabular}

Correlação de Pearson. Valores significativos ao nível de 1\% de probabilidade de erro.

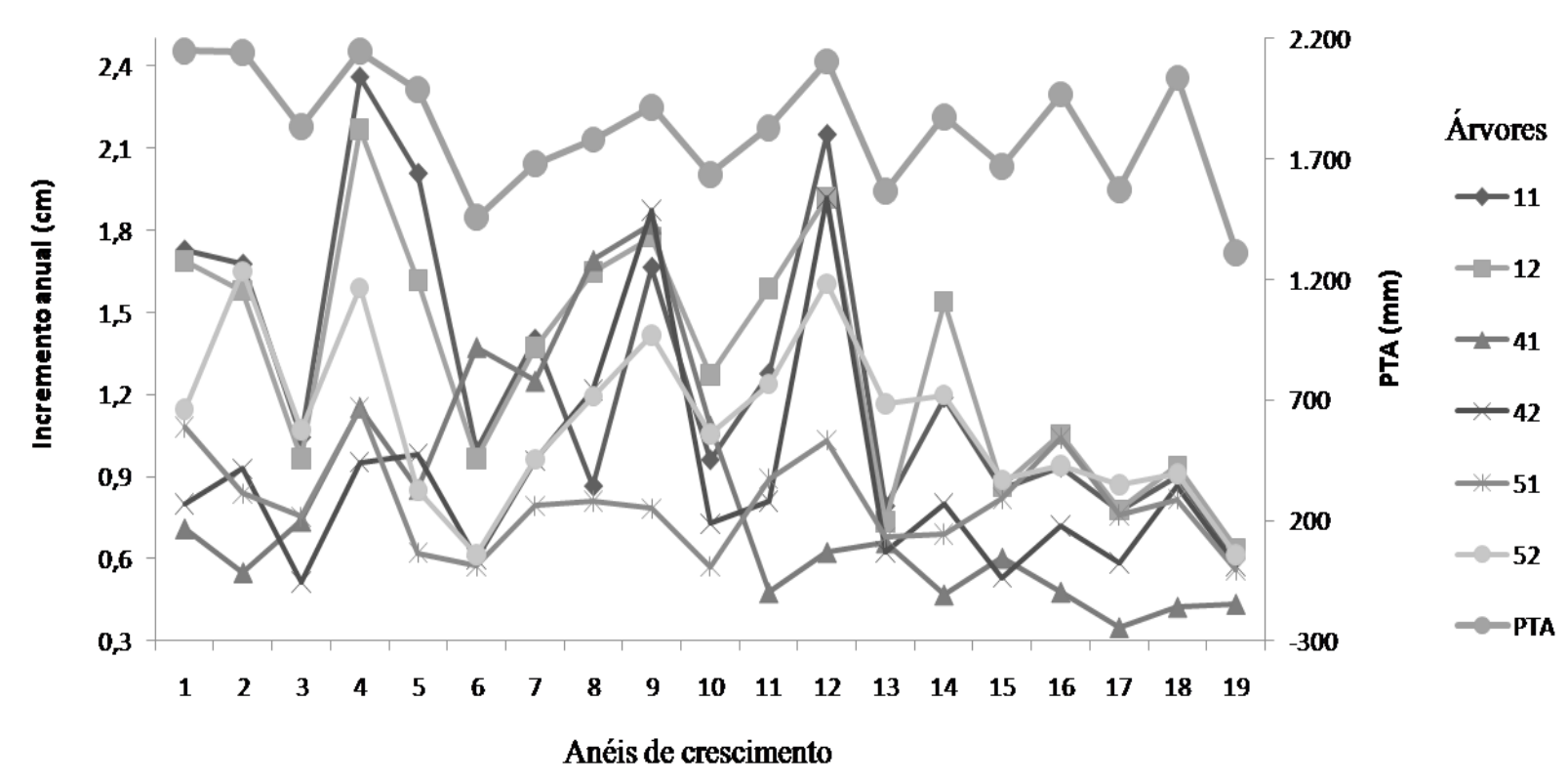

FIGURA 5: Valores de incrementos das árvores 1, 4 e 5 nas alturas 1 e 2 x precipitação total anual. FIGURE 5: Increments values for trees 1, 4 and 5 at heights 1 and $2 x$ total annual precipitation. 
Todas as correlações apresentadas foram positivas e significativas com probabilidade de 1\%. A árvore 1 apresentou maiores valores de correlação, com 0.7449 na altura 1 e 0.7275 na altura 2, entre precipitação e o incremento anual. Seguida pela árvore 5 com valores de correlação de 0.6854 na altura 1 e 0.7245 na altura 2, e pela árvore 4 que apresentou os menores valores de correlação de 0.5552 na altura 1 e 0.4698 na altura 2 . O fato das correlações mostrarem-se positivas indica que, quando um ano apresentar maior volume de chuvas, provavelmente maiores serão os valores de incremento dessas árvores (crescimento será favorecido). Ou seja, uma maior quantidade de água no solo aumenta as condições favoráveis ao crescimento da planta. Segundo Souza et al. (2006), os efeitos diretos e indiretos da deficiência de água no crescimento das árvores irá influenciar na relação da disponibilidade hídrica do solo e a produtividade florestal. Jacoby (1989), estudando o crescimento radial da teca (Tectona grandis L. f.) na Índia e em Java, pode concluir que este foi influenciado pela quantidade de chuva durante a estação seca em ambas as localidades. Mattos et al. (2004), encontraram correlação positiva entre o incremento radial e a precipitação pluviométrica para discos de 1 a $5 \mathrm{~m}$ de altura de árvores de Tabebuia heptaphylla proveniente do Pantanal Mato-Grossense. Brienen e Zuidema (2005) também encontraram relação positiva entre os anéis de crescimento e precipitação em certos períodos do ano para seis espécies tropicais da Amazônia boliviana.

Liphschitz (1995) fez observações semelhantes a estas para Quercus ithaburensis e Olea europaea, onde pode concluir que um abastecimento desfavorável de água resultava em baixas taxas de atividade cambial e madeiras com anéis porosos, enquanto um amplo abastecimento de água resultava em períodos mais longos de atividade cambial e porosidade difusa na madeira.

Ferraz (1993) constatou que a dependência da precipitação para a produção de matéria seca em eucaliptos é evidente. Comprovando para estas amostras a hipótese básica da dendroclimatologia, ou seja, a possibilidade de extrair a influência das variáveis climáticas, neste caso a precipitação pluviométrica, nas camadas de crescimento.

No ano de 1998, correspondente ao anel de número 13 (Figura 5), notou-se um menor incremento anual em diâmetro das árvores em relação ao ano anterior e posterior a este. Esse ano corresponde, na literatura, como ano subsequente ao de ocorrência do fenômeno El Niño. Callado e Guimarães (2010) estudando as prováveis causas da mortalidade da espécie Schizolobium parahyba na Ilha Grande, pertencente ao estado do Rio de Janeiro, diagnosticaram que os anéis de crescimento formados nos anos de 1998 e 1999 foram mais estreitos que dos outros anos avaliados. Segundo os mesmos autores, esses anéis estreitos foram formados sob a influência do evento El Niño dos anos de 1997-1998, considerado o mais importante do século, segundo a cronologia de intensidade, e o de maior influência na região sudeste do Brasil, originando drásticas anomalias na distribuição de chuvas (RAO et al. 2001, MINUZZI et al. 2005).

\section{CONCLUSÕES}

A técnica de densitometria de raios $\mathrm{X}$ possibilitou a análise precisa das variações radiais da densidade do lenho de árvores de Eucalyptus grandis. Os resultados deste estudo permitem concluir que os perfis diametrais indicaram um aumento da densidade do lenho no sentido medulacâmbio do tronco das árvores de Eucalyptus grandis. A variação radial da densidade indicou, da mesma forma, que as regiões interna e externa do tronco das árvores de eucalipto podem apresentar lenho de propriedades anatômicas e físicas diferenciadas. Em três árvores foi possível, através dos perfis radiais de densidade, delimitar os anéis anuais da madeira de Eucalyptus grandis. Os incrementos radiais anuais destas árvores, ao serem correlacionados com os totais anuais de precipitação local apresentaram correlação positiva ao nível significativo de $1 \%$. Foi observado, de uma forma geral, que quanto maior o volume de chuvas no ano, maiores serão os valores de incremento dessas árvores, e o crescimento será favorecido. Os resultados demonstram que a análise da largura dos anéis de crescimento obtidos pela densitometria de raios $\mathrm{X}$ pode ser usada aplicada com sucesso em árvores dos trópicos e é um método promissor para pesquisa em várias disciplinas. Porém para a aplicação da dendroclimatologia, realizar uma reconstrução climática local através dos anéis de crescimento, mais estudos devem ser feitos e um maior número de árvores com cronologias maiores devem ser analisado para gerar dados mais precisos.

\section{AGRADECIMENTOS}

Ao Conselho Nacional de Desenvolvimento

Ci. Fl., v. 21, n. 3, jul.-set., 2011 
Científico e Tecnológico (CNPq) pelo financiamento do projeto de pesquisa.

À Empresa QUINVALE Florestal Ltda. pelo material utilizado na realização estudo.

A todos do Laboratório de Anéis de Crescimento e Densitometria de Raios X do Departamento de Ciências Florestais da ESALQ/USP.

Ao Decanato de Pesquisa e Pós-Graduação da Universidade Federal Rural do Rio de Janeiro pelo apoio financeiro possibilitando a ida à Piracicaba-SP.

\section{REFERÊNCIAS BIBLIOGRÁFICAS}

AMARAL, A. C. B. Implantação da metodologia de densitometria de raios X em madeira. 1990. 109 f. Dissertação (Mestrado em Ciências Florestais) - Escola Superior de Agricultura "Luiz de Queiroz", Piracicaba, 1990.

BRIENEN, R. J. W.; ZUIDEMA, P. A. Relating tree growth to rainfall in Bolivian rain forests: a test for six species using tree ring analysis. Oecologia, v. 146, p. 1-12, 2005.

CALlADO, C. H.; GUIMARÃES, R. C. Estudo dos anéis de crescimento de Schizolobium parahyba (Leguminosae: Caesalpinioideae) após episódio de mortalidade em Ilha Grande, Rio de Janeiro. Rev. Bras. Bot., São Paulo, v. 33, n. 1, p. 85-91, 2010.

COUTINHO, A. P. Qualidade do carvão vegetal correlacionada com as características da madeira de Eucalyptus saligna e temperatura de carbonização. 1984. 79 f. Dissertação (Mestrado em Ciências Florestais)-Escola Superior de Agricultura "Luiz de Queiroz", Piracicaba, 1984.

COUTINHO, J. L. B. et al. Avaliação do comportamento de espécies de Eucalyptus spp. na Zona da Mata Pernambucana. I: Resultados do primeiro ano - 2001. Rev. Árvore, Viçosa, v. 28, n. 6, p. 771-775, 2004.

ENCINAS, J. I.; SILVA, G. F.; PINTO, J. R. R. Idade e crescimento das árvores. Brasília: Comunicações Técnicas Florestais, UNB, 2005. 47 p.

FERRAZ, E. S. B. Anéis de crescimento e clima em Eucalyptus. Silvicultura, São Paulo, v. 8, n. 32, p. 821-822, 1993.

FERREIRA, M. Estudo da variação da densidade básica da madeira de povoamentos de Eucalyptus grandis Hill ex Maiden. 1970. 62 f. Tese (Livre Docência)-Escola Superior de Agricultura "Luiz de Queiroz", Piracicaba, 1970.

FOELKEL, C. E. B. et al. Variabilidade radial da madeira de Eucalyptus grandis. Silvicultura, São
Paulo, v. 8, p. 782-791, 1987.

JACOBY, G. G. Overview of tree ring analyses in tropical regions. IAWA, Leiden, n. 10, p. 99-108, 1989.

LIPHSCHITZ, N. Ecological wood anatomy: changes in xylem structure in Israeli trees. Beijing, Wu Shuming, Wood anatomy research, International Academic Publishers, 1995. 11-15 p.

MATTOS, P. P.; SEITZ, R. A.; SALIS, S. M. Potencial dendroecológico de Tabebuia heptaphylla (Vell.) Toledo. Boletim de Pesquisa Florestal, Colombo, n. 48, p. 93-103, 2004.

MINUZZI, R. B. et al. El Niño: ocorrência e duração dos veranicos do Estado de Minas Gerais. Rev. Bras. de Eng. Agr. e Amb., Campina Grande, v. 9, p. 364-371, 2005.

PALERMO, G. P. M. Parâmetros de qualidade da madeira de Pinus elliottii Engelm. de diferentes idades. 2003. 138 f. Dissertação (Mestrado em Ciências Ambientais e Florestais)-Universidade Federal Rural do Rio de Janeiro, Rio de Janeiro, 2003.

PANSHIN, A. J.; ZEEUW, C. E. Textbook of wood technology. New York: McGraw-Hill, 1970. 722 p. OLIVEIRA, J. T. de; TOMAZELLO FILHO, M.; SILVA, J. C. Resistência natural da madeira de sete espécies de eucalipto ao apodrecimento. Revista Árvore, Viçosa, v. 29, n. 6, p. 993-998, 2005.

RAO, V. B.; SANTO, C. E.; FRANCHITO, S. H. A diagnosis of rainfall over South America during 1997/98 El Niño event. Part I: validation of NCEPNCAR reanalysis of rainfall data. Journal of Climate, Boston, v. 15, p. 502-511. 2001.

REZENDE, M. A.; FERRAZ, E. S. B. Densidade anual da madeira de Eucalyptus grandis. IPEF, Piracicaba, n. 30, p. 37-41, 1985.

REZENDE, M. A.; FERRAZ, E. S. B. Incrementos anuais de volume, massa e idade ideal de corte para Eucalyptus grandis. IPEF, Piracicaba, n. 32, p. 4348, 1986.

REZENDE, M. A.; SAGLIETTI, J. R.; CHAVES, R. Variação da massa específica da madeira de Eucalyptus grandis aos 8 anos de idade em função de diferentes níveis de produtividade. Revista Scientia Forestalis, Piracicaba, n. 53, p. 71-78, 1998.

RASPOPOV, O. M.; DERGACHEV, V. A.; KOLSTRÖM, T. Hale Cyclicity of Solar Activity and Its Relation to Climate Variability. Journal Solar Physics, The Netherlands, v. 224, n. 1-2, p. 455-463, 2004.

RIBEIRO, F. A.; ZANI FILHO, J. Variação da densidade básica da madeira em espécies/ 
procedências de Eucalyptus spp. IPEF, Piracicaba, v. 46, p. 76-85, 1993.

RIGOZO, N. R. et al. Prediction of solar activity on the basis of spectral characteristics of sunspot number. Annales Geophysicae, Göttingen, v. 22, n. 6, p. 2239-2243, 2004.

ROQUE, R. M. Variação da anatomia e da densidade básica da madeira de Gmelina arborea (Roxb.), em diferentes condições de clima e manejo na Costa Rica. 2005. 202 f. Tese (Doutorado em Recursos Florestais)-Escola Superior de Agricultura "Luiz de Queiroz", Piracicaba, 2005.

SILVA, J. C. Caracterização da madeira de Eucalyptus grandis Hill ex. Maiden, de diferentes idades, visando a sua utilização na indústria moveleira. 2002. 160 f. Tese (Doutorado em Ciências Florestais) - Universidade Federal do Paraná, Curitiba, 2002.

SOUZA, M. J. H. et al. Disponibilidade hídrica do solo e produtividade do eucalipto em três regiões da
Bacia do Rio Doce. Revista Árvore, Viçosa, v. 30, n.3, p. 399-410, 2006.

TOMAZELLO FILHO, M. Variação radial da densidade básica e da estrutura anatômica da madeira do Eucalyptus saligna e Eucalyptus grandis. IPEF, Piracicaba, n. 29, p. 37-45, 1985.

TOMAZELLO FILHO, M. Efeito da irrigação e fertilização nas propriedades do lenho de árvores de Eucalyptus grandis $\mathbf{x}$ urophylla. 2006. $146 \mathrm{f}$. Tese (Livre Docência em Ciência e Tecnologia de Madeira)-Escola Superior de Agricultura "Luiz de Queiroz", Piracicaba, 2006.

TROVATI, L. R.; FERRAZ, E. S. B. Influência da precipitação e da temperatura na densidade dos anéis de crescimento de Pinus oocarpa. IPEF, Piracicaba, n. 26, p. 31-36, 1984.

VITAL, B. R.; DELLA LUCIA, R. M. Efeito do espaçamento na produção em peso e na qualidade da madeira de Eucalyptus grandis e Eucalyptus urophylla aos 52 meses de idade. Revista Árvore, Viçosa, v. 11, n. 2, p. 132-145, 1987. 